\title{
Opportunities of Federated Learning in Connected, Cooperative and Automated Industrial Systems
}

\author{
Stefano Savazzi, Monica Nicoli, Mehdi Bennis, Sanaz Kianoush, Luca Barbieri
}

\begin{abstract}
Next-generation autonomous and networked industrial systems (i.e., robots, vehicles, drones) have driven advances in ultra-reliable, low latency communications (URLLC) and computing. These networked multi-agent systems require fast, communication-efficient and distributed machine learning (ML) to provide mission critical control functionalities. Distributed ML techniques, including federated learning (FL), represent a mushrooming multidisciplinary research area weaving in sensing, communication and learning. FL enables continual model training in distributed wireless systems: rather than fusing raw data samples at a centralized server, FL leverages a cooperative fusion approach where networked agents, connected via URLLC, act as distributed learners that periodically exchange their locally trained model parameters. This article explores emerging opportunities of FL for the next-generation networked industrial systems. Open problems are discussed, focusing on cooperative driving in connected automated vehicles and collaborative robotics in smart manufacturing.
\end{abstract}

\section{INTRODUCTION}

The rapid transformation of industrial systems, driven by the digitization and $5 \mathrm{G}$ communication evolution, has led to extensive research initiatives on manufacturing and automotive verticals. These include, for example, Industry 4.0 (I4.0), at European level, the European Factories of the Future Research Association (EFFRA, effra.eu), the 5G Alliance for Connected Industries and Automation (5g-acia.org) and the 5G Automotive Association (5gaa.org). The envisioned smart industrial systems rely on networked machines with increasing level of intelligence and autonomy, moving far beyond traditional lowcost, low-power sensors. According to industrial requirements, such machines are required to support:

1) autonomous and adaptive decision making in dynamic situations with mobile operators/equipment, device-less humanmachine interfaces and time-varying environments;

(C)2021 IEEE. Personal use of this material is permitted. Permission from IEEE must be obtained for all other uses, in any current or future media, including reprinting/republishing this material for advertising or promotional purposes, creating new collective works, for resale or redistribution to servers or lists, or reuse of any copyrighted component of this work in other works.

S. Savazzi and S. Kianoush are with the Institute of Electronics, Computer and Telecommunication Engineering (IEIIT) of Consiglio Nazionale delle Ricerche (CNR), Milano, Italy.

M. Nicoli and L. Barbieri are with Politecnico di Milano DIG and DEIB department, Milano, Italy.

M. Bennis is with the Centre for Wireless Communications, University of Oulu, Finland.

The paper has been accepted for publication in the IEEE Communications Magazine. The work is partially supported by the European Project CHISTERA RadioSense (Big Data and process modelling for the Smart Industry BDSI) funded by MUR and by the Project BASE5G (Broadband InterfAces and services for Smart Environments enabled by $5 \mathrm{G}$ technologies), funded by the Italian Lombardy Regional Government under the grant POR-FESR 2014-2020 ID 1155850. The current arXiv contains an additional Appendix that describes the MIMO radar dataset for the setup of Fig. 5. Sample dataset published on IEEE Data Port http://dx.doi.org/10.21227/0wmc-hq36
2) big-data-driven training of large-size machine learning (ML) models for decision-making;

3) ultra-reliable and low-latency communications (URLLC) [1] for mission-critical device-to-device (D2D) operations.

Networked and cooperative intelligent machines have recently opened new research opportunities that target the integration of distributed ML tools with sensing, communication and decision operations. Cross-fertilization of these components is crucial to enable challenging collaborative tasks in terms of safety, reliability, scalability and latency.

Among distributed ML techniques, federated learning (FL) [2], [3] has been emerging for model training in decentralized wireless systems. Model parameters, namely weights and biases in deep neural network (DNN) layers, are optimized collectively by cooperation of interconnected devices, acting as distributed learners. In contrast to conventional edge-cloud ML, FL does not require to send local training data to the server, which may be infeasible in mission critical settings with extremely low latency and data privacy constraints.

The most popular FL implementation, namely federated averaging [2], alternates between the computation of a local model at each device and a round of communication with the server for learning of a global model. Local models are typically obtained by minimizing a local loss function via Stochastic Gradient Descent (SGD) steps [3], using local training examples and target values.

Federated averaging is privacy-preserving by design, as it keeps the training data on-device. However, it still leverages the server-client architecture, which might not be robust to data poisoning attacks and scalability needs. Overcoming this issue mandates moving towards fully decentralized FL solutions relying solely on local processing and cooperation among end machines. As shown in Fig. 1, the device sends its local ML model parameters to neighbors and receives in return the corresponding updates. Next, it improves its local parameters by fusing the received contributions. This procedure continues until convergence.

The article addresses the opportunities of emerging distributed FL tools specifically tailored for systems characterized by autonomous industrial components (vehicles, robots). FL is first proposed as an integral part of the sensing-decisionaction loop. Next, novel decentralized FL tools and emerging research challenges are highlighted. The potential of FL is further elaborated with considerations primarily given to mission critical control operations in the field of cooperative automated vehicles and densely interconnected robots. Analysis with real data on a practical usage scenario reveals FL as a promising tool underpinned by URLLC communications. 


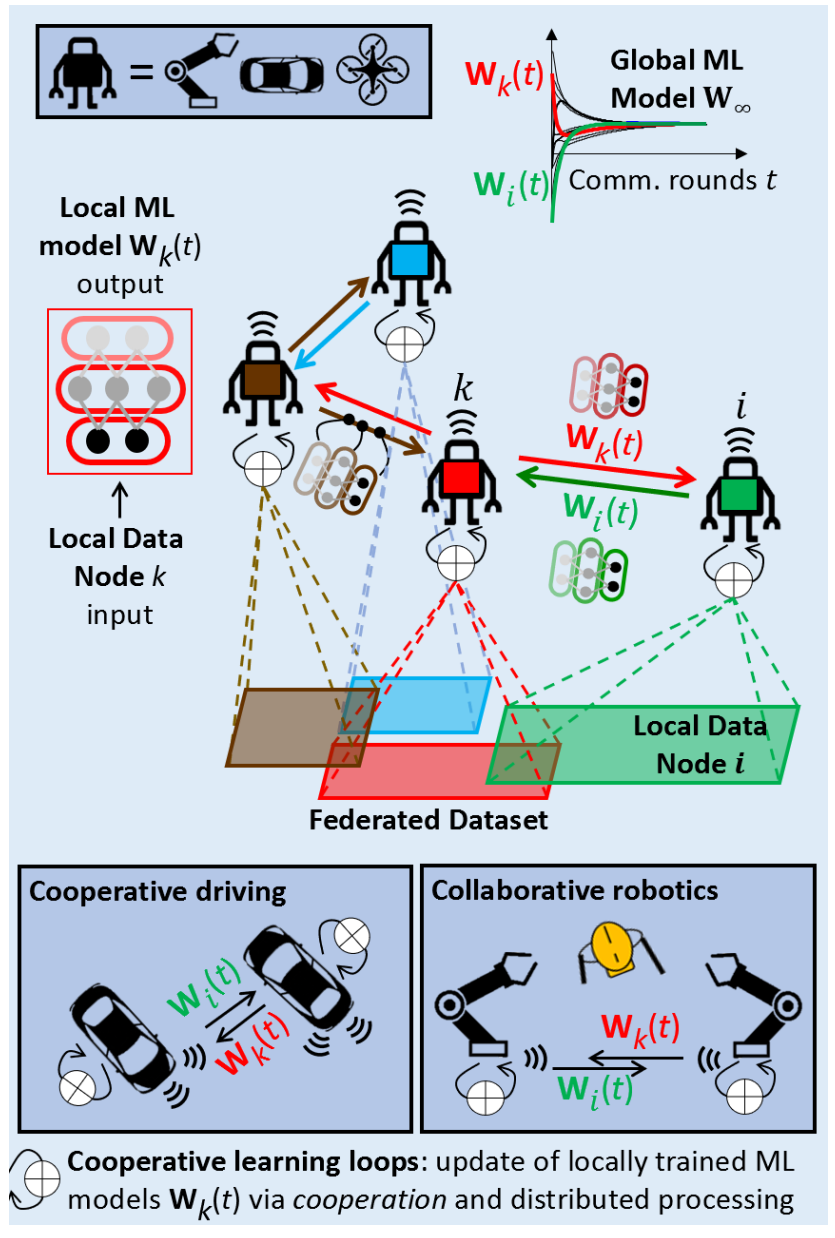

Fig. 1. Decentralized learning with federated datasets. Examples in mission critical control applications: collaborative industrial robotics and cooperative automated vehicles.

\section{SENSING, DECENTRALIZED LEARNING AND COMMUNICATION CO-DESIGN}

Decentralized FL solutions have great potential for industrial 5G and beyond 5G (B5G) verticals. In automated industrial processes, decentralized FL imbues intelligence directly into the end machines, which become smart cooperative agents. Fig. 2 depicts a schematic of a cooperative and automated multi-agent industrial system. It consists of connected machines performing collaborative tasks, and integrating ML model training within the sensing-decision-action loop. The ML model outputs might be scenario-dependent predictions of a physical process, or rather value functions to be used for policy improvements (i.e., reinforcement learning). Outputs are fed to the machine controller for local decisions or actuations. Training of the ML model calls for highly efficient knowledge discovery operations based on the overall training data collected by all the machines performing the same task (federated dataset), rather than local data only. Recent advancements of FL constitute a first but significant step towards collaborative learning and, particularly, the understanding of how ML could be distributed over networked devices, without centralized orchestration. Collaborative learning underpins local decisions and allows the networked machines to augment their ML

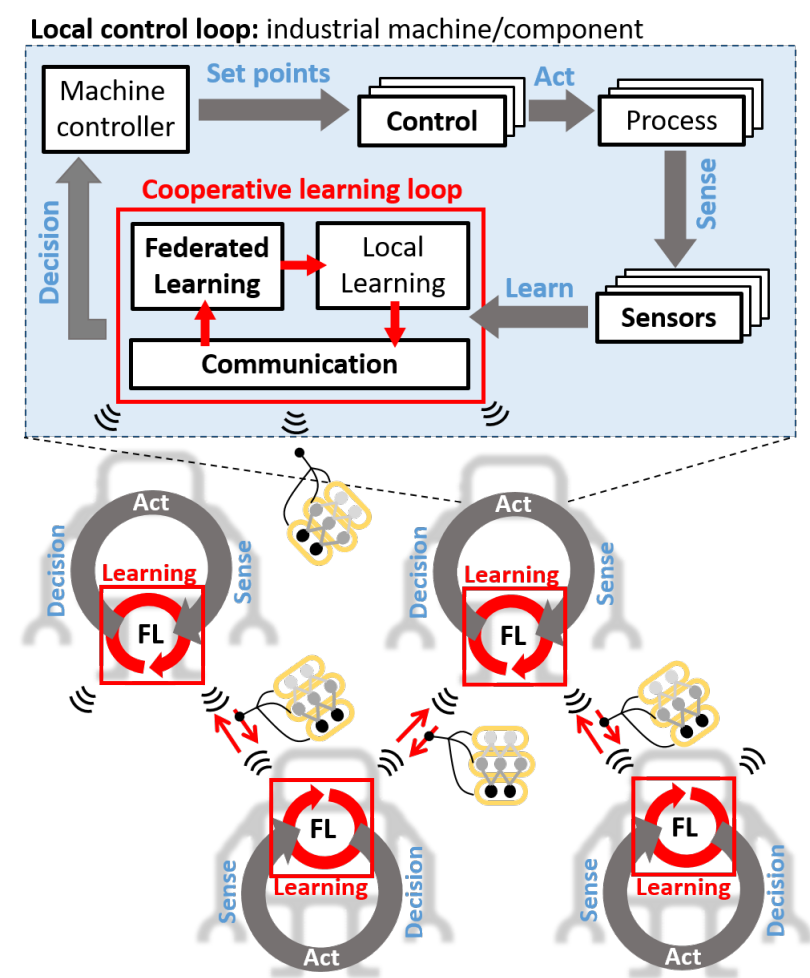

Fig. 2. Decentralized FL in the sensing-decision-action loop.

model by sharing ego knowledge. As part of the sensingdecision-action loop, emerging FL tools are expected to target three fundamental challenges:

1) get over the restrictions of server-client architectures and movements of large, unstructured, raw datasets over D2D wireless links, in favor of (typically) sparse ML model parameters exchange;

2) optimally balance opportunistic (ego) learning based on local training data, and collaborative learning (leveraging neighbor's experience), with the goal of steering convergence;

3) learn and re-train continuously over URLLC links to adapt to changes in the data distribution, environment, process or situation.

\section{DeCEntralized FL: EMERGING TRENDS}

Decentralized FL alternates the mutual exchange of local ML models $\mathbf{W}_{k}(t)$ with the on-device minimization of a local loss function. The goal is to promote convergence to a global model $\mathbf{W}_{\infty}$ that minimizes a global loss, decomposed into the sum of local losses. Fully distributed FL implementations run on top of D2D networks characterized by arbitrary connectivity graphs. The collaborative learning is based on Decentralized Stochastic Gradient Descent (DSGD), which guarantees convergence under strong convexity assumptions of local loss functions [4] and networks with doubly stochastic adjacency matrix [3]. DSGD alternates on-device SGD steps to obtain $\mathbf{W}_{k}(t)$, with the mutual exchange of model parameters to steer convergence. Mutual exchange is regulated by gossip [5], consensus or diffusion algorithms [6]-[7]. 


\section{A. Consensus, diffusion and gradient negotiations}

In consensus based approaches, [5]-[6], federated nodes exchange local ML model parameters and update them sequentially by distributed averaging. For the mutual exchange of models, nodes might select random, time-varying or optimized [8]-[12] partners. In gossipgrad [5], the selection policy creates a virtual network where each cooperating agent is connected to, typically, two other nodes. Convergence time and loss are ruled by the specific choice of model update operations. Some updating strategies favor fast convergence [6] while others target model accuracy [12]. Considering DNN model training, the neural network layers are also typically learned separately and independently. Therefore, on-device optimization and networking phases can run in parallel. Communication overhead scales linearly with the model size and the number of cooperating agents. However, non-independent-identicallydistributed (non-IID) federated datasets and large model size penalize the rate of convergence [3].

Diffusion strategies incorporate a gradient negotiation phase, described in Fig. 3, where devices exchange the information about how neighbor models should be adjusted considering local data. Diffusion virtually expands the local training data-set, and boosts the convergence compared with gossip. Gradient exchange strategies are often regulated by request-reply negotiation stages between neighbors [7]. In the example of Fig. 3, the local model $i, \mathbf{W}_{i}(t=1)$, is sent to the neighbor $k$ at time $t=1$ to start a negotiation. The received model is used by the neighbor $k$ to compute a gradient vector $\nabla \mathbf{W}$ using the local loss. Both the gradient vector and the local model are then fed-back to the device $i$, that started the negotiation $(t=2)$. On-device optimization $(t=3)$ finally adjusts the local model by combining the gradients obtained from local loss with those received from the neighbor (combine-and-adapt [7]). In [6] the negotiation resorts to a two-stage asynchronous procedure: convergence speed improves at the cost of larger communication overhead. Exploring this trade off is currently an open challenge.

In addition to the above tools, distributed ledger technologies can also be applied to decentralized training [11] by validating clients model updates via a series of validation steps (Proof-of-Work, or others). As a result, decentralized FL is transformed into a market of expert model training nodes and validators. The development of robust FL designs against data poisoning and adversarial manipulations is still an open problem.

\section{B. Improving communication efficiency}

Reliable and low-latency D2D communications serve as the backbone for distributed FL computations. Transmission of model parameters must be extremely reliable with packet error rate down to $10^{-8}$ to prevent frequent retransmissions penalizing the convergence rate [6]. Transmission time intervals (TTI) should be aligned with the data dynamics and the computation times required to perform SGD and model adaptation, targeting $5 \mathrm{~ms}$ and below [1]. The model size also affects communication design choices: DNN models often

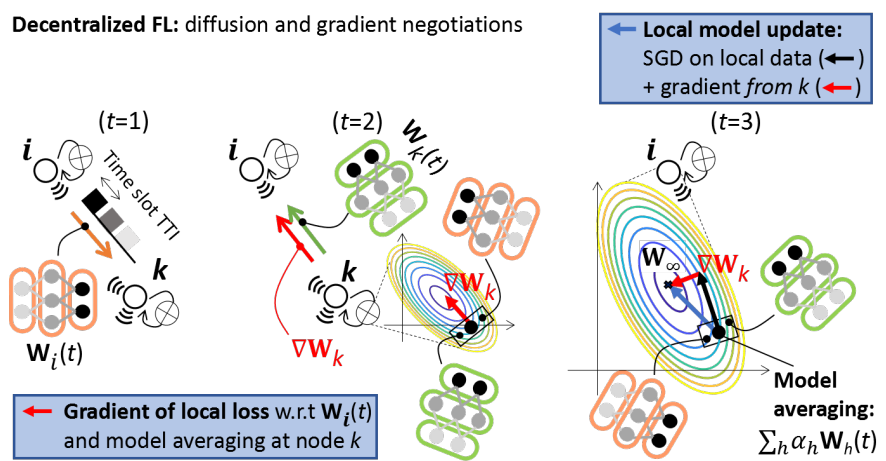

Fig. 3. Decentralized FL with diffusion: communication, gradient negotiations $(t=1,2)$ and computing rounds $(t=3)$.

used in industrial applications contain $>15 \mathrm{k}$ parameters per layer, usually extremely sparse.

Although vanilla FL assumed noiseless or rate-limited communications [3], recently proposed digital and analog designs quantify the effects of intermittent communications, time-varying fading and interference. Digital implementations of FL require each device to communicate with peers over half-duplex links via time scheduled wireless access. Popular solutions to limit the model size, and thus the communication overhead, are quantization, sparsification and distillation [8]. Sparsifying operators select a subset of informative ML parameters, often improving also the model generalization.

More recently, analog FL and hybrid analog-digital implementations have been proposed for fast and synchronous model averaging [4]. Analog FL gets over the restrictions of time scheduled access as it exploits the superposition property of dense wireless transmissions when averaging the neighbor models. Each device receives the superposition of the ML models simultaneously transmitted by the neighborhood. When ML parameters are sparse and sent as uncoded/uncompressed, Lasso type recovery can be adopted for decoding and reconstruction [9].

Besides low-level communications, optimization of resource allocation has been considered to find the best trade-off between on-device computation (SGD updates) and wireless channel use for parameters exchange [10]. Optimized medium access control can also substantially reduce the FL loss [12]: for example, scheduling should reward devices having high quality data compared to those possessing few, or redundant samples [11].

\section{Emerging research challenges}

The roll-out of new decentralized communication paradigms in $\mathrm{B} 5 \mathrm{G}$ is expected to bridge the gap between deep learning and wireless networking research, raising at the same time unprecedented challenges. For example, current FL designs generally ignore the underlying dynamics of the network graph, the presence of intermittent communication links or weakly connected components [3]. Research in this direction should focus on balancing local data collection, model adaptations and cooperation from selected agents. Digital, analog or hybrid implementations should be considered based on devices' mobility, model size, computational and bandwidth 


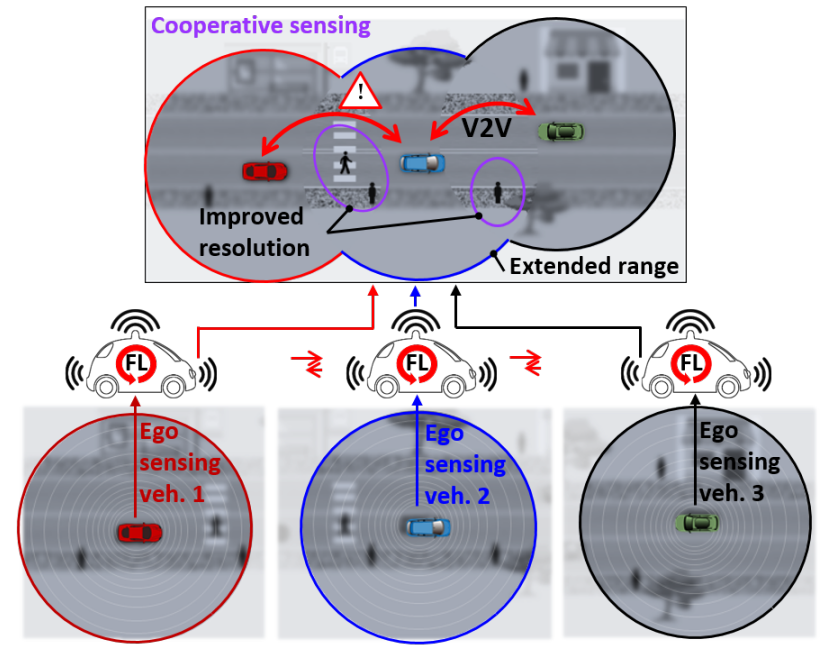

Fig. 4. Distributed FL for cooperative perception by real-time fusion of imaging data at different vehicles.

resources, as well as connectivity quality. Learning an optimal policy for graph (and/or neighbor) selection while training the ML model, namely learning simultaneously the network graph and the model, is also a promising direction.

Automated systems are often characterized by heterogeneous devices performing distinct but related tasks. Collaborative learning of multiple functions, namely multitask learning [13], will have a relevant impact on several industrial applications. Understanding when cooperation, in the form of consensus, or any decentralized agreement protocol, can better steer convergence compared with opportunistic or ego behaviors is an open problem of wide interest. The analysis must also take into consideration the cost of distributed computations, the federated data distribution and the network designs for URLLC.

Using the I4.0 vision and emerging B5G verticals as guidelines, in what follows opportunities of FL are highlighted for the needs of selected mission critical control applications, namely cooperative automated driving and collaborative robotics.

\section{Distributed INTELLIGENCE FOR COOPERATIVE AUTOMATED DRIVING}

Vehicular URLLC leverage B5G connectivity to enable flexible vehicle-to-everything (V2X) interactions with road infrastructure (V2I) and other vehicles (V2V). Distributed ML over V2X networks will play a central role in cooperative intelligent transportation system (C-ITS), enabling level 4-5 cooperative automated driving functionalities [14]. Cooperative automated vehicles share maps of the driving environment (Fig. 4) using V2X URLLC to extend the range and resolution of their ego imaging sensors (radar, camera, lidar). Beside improving detection and localization of safety-related events, vehicles can also negotiate the maneuvering and synchronize to a common mobility pattern, forming tight autonomousdriving convoys and increasing traffic efficiency. All the above scenarios are characterized by time-critical functions that must be implemented on a closer-to-the-ground cloud, with part of the cooperative computational tasks performed locally, by pushing intelligence into the vehicles rather than on the mobile edge cloud (MEC) [14].

Decentralized FL techniques [6] are promising solutions for these time-sensitive applications. They require the vehicles to transmit smaller models which can be aggregated at the road side unit (RSU) or by vehicles via ultra-low latency (3 ms) and highly reliable $\left(10^{-5}\right) \mathrm{V} 2 \mathrm{X}$ connectivity. The exchange of local ML model parameters, rather than raw data, is expected to decrease the learning time, allowing to quickly react to unexpected events and take safety-critical decisions. Domain-specific FL designs must also account for intermittent communications, time-varying network graphs and non-IID training datasets changing quickly over time, thus evolving according to vehicle motions, with speeds up to 250 $\mathrm{km} / \mathrm{h}$ (3GPP TR 22.886), and the surrounding environment. In such cases, a transitory phase is expected where vehicles with outdated, or partially trained models, will coexist with highly-automated ones and benefit from their cooperation. The federation with fully equipped vehicles will assist lower-level vehicles to get an augmented vision of the driving environment, even if equipped with less accurate sensors. Balancing between centralized and decentralized FL implementations for energy optimization is also a main issue. Besides cooperative driving, to improve safety and reliability, the vehicles need also to learn the network latency in a distributed manner [1], whereby decentralized FL (and its variants) are instrumental. Open problems further relate to the tension between local and global models, and the impact of large numbers of vehicles.

Fig. 4 highlights a distributed ML-assisted cooperative perception task where vehicles fuse their sensor data in a decentralized implementation to get an extended vision of the environment. Fusing dynamics (i.e., global navigation satellite systems, GNSS, inertial measurement units, IMU) and imaging sensor data (i.e., lidar, camera, radar) from different vehicles improves the location sensing accuracy making real-time responses feasible. A main challenge is the association of unlabeled imaging measurements at different vehicles to jointly sensed features for cooperative simultaneous localization and mapping (SLAM). Large data volumes and computational complexity are also critical challenges. Decentralized FL is a promising candidate, as it is able to learn a common model for data association and fusion from local raw data, limiting the V2X exchange to model parameters.

\section{DeCENTRALIZED FL FOR COBOTS}

The development of collaborative robotics in advanced manufacturing environments (cobots) can be interpreted as parallel to autonomous driving. Standardized in the ISO/TS 15066, collaborative robot operations allow the machines (industrial manipulators, vehicles) installed in a shared workspace to move concurrently with human operators inside fenceless environments. Connected and collaborative robots are transforming industrial workspaces, such as assembly lines in the example of Fig. 5(a), and represent a challenging ground for the development of decentralized FL tools. First, robots operate in increasingly complex and time-varying environments. 
(a)

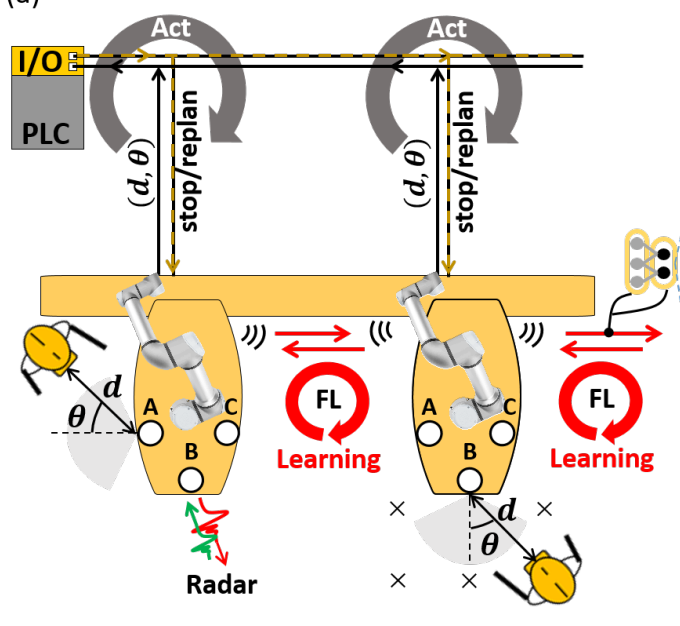

(b)

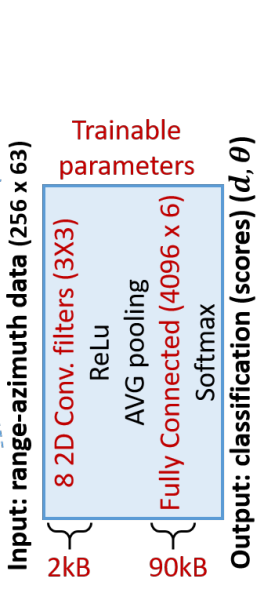

(b)
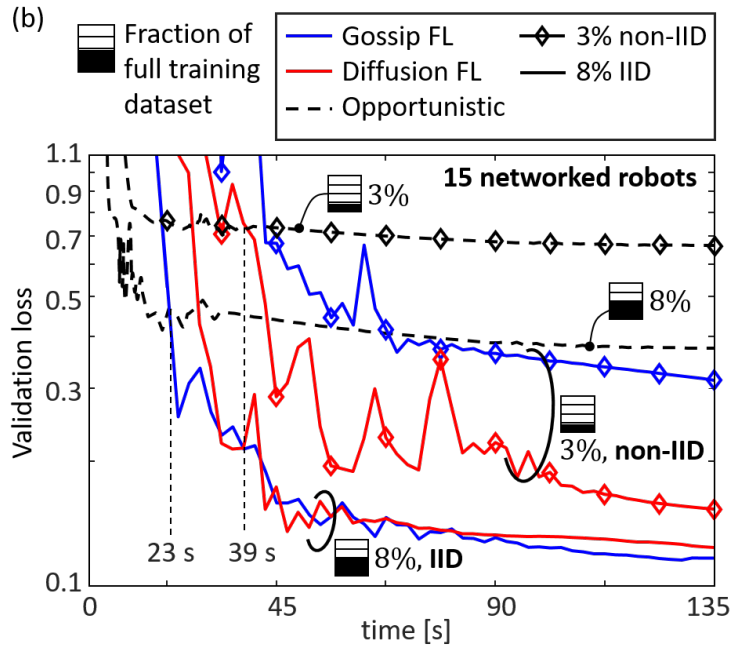

Fig. 5. (a) Decentralized FL setup in a cobot environment: frequency-modulated continuous wave (FMCW), multiple-input-multiple output (MIMO) radar locations (A,B,C) and ML model based classification of subject positions; (b) FL loss for gossip (blue) and diffusion (red) vs. time and over a D2D network of 15 robots. IID (no markers) and non-IID (diamond markers) federated datasets using the $8 \%$ and $3 \%$ of the full training dataset, respectively. D2D network with $\mathrm{TTI}=3 \mathrm{~ms}, \mathrm{BLER}=10^{-9}$. Dashed lines refer to the opportunistic learning case.

Network design, paired with distributed learning tools, must consider the problem of continual learning (and periodic retraining) over URLLC communication links to track variations of data dynamics caused by changes of the workflow process. Second, robots might perform distinct tasks, although these can be considered as strongly related, targeting a common manufacturing process (assembly or dis-assembly tasks) and efficient workspace sharing. In such cases, opportunistic (ego) and collaborative approaches should be carefully assessed. For example, FL can boost the model training for some tasks, such as vision functions, in common to many robots.

To shed light on some of the above challenges, we resort to a typical mission critical control problem in Industry 4.0 workspaces. The goal is to plan the motions of a robotic manipulator based on the information about the positions of human operators sharing the workspace. Operator locations are classified using a ML model, described in Fig. 5.a), that computes the human-robot distance $d$ and the direction of arrival (DOA) $\theta$ in real-time, using 6 regions of interest as target classes. During the on-line workflow, the ML model can be trained/updated continuously by decentralized FL and consensus (cooperative learning loop). The sensing-decision-action loop is supervised by an industry standard programmable logic controller (PLC) that makes decisions about robot emergency stop, or trajectory replanning [15], based on the information about subject positions. Decentralized FL uses D2D links, runs in parallel to the sensing-decision-action loop, and thus takes some load off of the PLC network, whose resources must be reserved for robot motion control. All the robots support vision functions implemented by 3 radars working in the $77-81 \mathrm{GHz}$ band with a field-of-view of $120 \mathrm{deg}$ each. Radars produce the raw data [15] that are fed into the ML model.

\section{RESULTS AND DISCUSSIONS}

The example of Fig. 5(b) analyses the performance of decentralized FL approaches compared with ego, i.e., oppor- tunistic, learning, considering the application case previously described. In particular, the robots might choose to combine in groups of 15 agents and implement FL over a D2D network, or rather act opportunistically, thus learning from local data only, disabling the D2D radio interface. The examples also highlight the impact of federated data distribution on convergence time and validation loss. When federated data is IID partitioned, each device uses $8 \%$ of the full training dataset of 900 samples. In the more challenging non-IID scenario, only the $3 \%$ of the data is used: batches contain examples for only 5 of the 6 output target classes, chosen randomly.

D2D communications are organized into consecutive frames and use time-division multiple access (TDMA) scheduling. Frames have payload $1 \mathrm{kB}$ and TTI of $3 \mathrm{~ms}$, in line with URLLC [1]. Notice that a frame drop (BLER is $10^{-9}$ ) causes the loss of a layer update, and an increase of the convergence time. FL and networking have been simulated on a virtual environment but using real data from the plant. The environment allows to deploy networked agents acting as virtual robots and learning over a configurable federated dataset (IID and nonIID).

The simulations of Fig. 5(b) quantify the average validation loss [1] experienced by the deployed agents versus time. This allows to assess the time required by the consensus rounds, as relevant for real-time implementation. Gossip and diffusion FL approaches, described previously, are evaluated over a kregular D2D network consisting of robots with 2 neighbors each. Implementation of gossip is based on [5]: on each round, the agents randomly choose a single neighbor from the neighborhood, exchange the local ML models and adapt them by averaging, followed by SGD on local data. Diffusion FL implements the gradient negotiations described in Fig. 3 on each round the agents now exchange the local ML models and the gradients $\nabla \mathbf{W}$ with a single neighbor, chosen again randomly. For both cases, the time span of an individual round consists of the transmission of the ML parameters 
in the assigned frames, the model adaptation and the SGD steps (30ms per round). Before D2D transmission, the ML model parameters are sparsified [9] to limit the communication overhead, measured in $\mathrm{kB}$ per round.

Diffusion FL boosts the model training for robots possessing few data (3\%) and insufficient examples (non-IID). Compared with ego learning, cooperation gives smaller loss after a training period of $39 \mathrm{~s}$, that is enough to track workflow changes. Diffusion needs considerable communication overhead (220 $\mathrm{kB}$ per round) due to gradients exchange. Convergence time thus increases with the network size. Notice that small but sudden increases of the loss are observed on some rounds when averaging the neighbor models. These effects are more visible when the local models are trained with non-IID data and can be mitigated by learning rate optimization [6]. Gossip requires twice lower number of frames (92 $\mathrm{kB}$ per round) but it is less effective over non-IID data. However, it is still useful for refining models trained with IID data as it improves ego approaches after $23 \mathrm{~s}$. Opportunistic learning converges fast as it does not utilize D2D communications, but experiences a large loss: it is a viable solution only for agents possessing enough data and performing specialized tasks that do not require much re-training.

\section{CONCLUSIONS AND FUTURE DIRECTIONS}

In this article we explored opportunities and applications of FL in networked and automated industrial systems, underpinned by D2D wireless communications. Open problems and challenges have been discussed, focusing on manufacturing and automotive B5G verticals. Decentralized FL enables the cooperative learning of ML models. It can be seamlessly integrated into the application dependent sensing-decision-action loop within each automated entity to improve knowledge discovery operations. Learning and re-training continuously to follow the changing dynamics of the environment have a profound impact on the networking co-design, capitalizing on model sparsity and superposition properties of the wireless links.

Future research is expected to target increasingly complex mobile environments characterized by heterogeneous devices cooperating to learn distinct, but related, functions. The choice between opportunistic and cooperative behaviors will largely depend on the URLLC design. Emerging FL tools are promising in cooperative automated driving, leveraging V2X interactions, and in collaborative robotics for distributed learning in complex and dynamic workflows.

\section{APPENDIX: FederATED DATA AND ML MODEL}

As shown in Fig. 5, the robots are equipped with 3 MultipleInput-Multiple-Output (MIMO) Frequency Modulated Continuous Wave (FMCW) radars, working in the $77 \mathrm{GHz}$ band. Radars implement a Time-Division (TD) MIMO system with 2 transmit and 4 receive antennas each, and a field-of-view of $120 \mathrm{deg}$. During the on-line workflow, the distance $d$ and DOA $\theta$ information are classified by the agents using a trained ML model. The ML model is here trained to classify 6 potential HR collaborative situations characterized by different
HR distances and DOA ranges, corresponding to safe or unsafe conditions.

Based on the above setup, a simplified database for testing, is available in the repository/http://dx.doi.org/10.21227/0wmchq36 The database contains 4 main data structures, detailed as follows:

i) mmwave_data_test has dimension $900 \times 256 \times 63$. Contains 900 FFT range-azimuth measurements of size $256 \times 63$ : 256-point range samples corresponding to a max range of $11 \mathrm{~m}$ (min range of $0.5 \mathrm{~m}$ ) and 63 angle bins, corresponding to AOA ranging from -75 to +75 degree. Data are used for testing (validation database). The corresponding labels are in label_test. Each label (from 0 to 5) corresponds to one of the 6 positions (from 1 to 6 ) of the operator as detailed in the Fig. $5 \mathrm{~b}$.

ii) mmwave_data_train has dimension $900 \times 256 \times 63$. Contains 900 FFT range-azimuth measurements used for training. The corresponding labels are in label_train.

iii) label_test with dimension $900 \times 1$, contains the true labels for test data (mmwave_data_test), namely classes (true labels) correspond to integers from 0 to 5 .

iv) label_train with dimension $900 \times 1$, contains the true labels for train data (mmwave_data_train), namely classes (true labels) correspond to integers from 0 to 5 .

The implemented ML model takes as input the raw rangeazimuth data (after background subtraction) of size $256 \times 63$ from the radars. As shown in Fig. 5 a, it consists of 2 trainable neural network layers of 25.000 parameters. Decentralized FL uses gossip or diffusion methods (described previously) with SGD step size $\mu_{t}=0.025$.

\section{REFERENCES}

[1] M. Bennis, et al. "Ultrareliable and Low-Latency Wireless Communication: Tail, Risk, and Scale," Proc. of the IEEE, vol. 106, no. 10, pp. 1834-1853, Oct. 2018.

[2] Konečný J., et al. "Federated optimization: Distributed machine learning for on-device intelligence," CoRR, 2016. [Online]. Available: http://arxiv.org/abs/ 1610.02527.

[3] P Kairouz, et al., "Advances and open problems in federated learning,", [Online]. Available: https://arxiv.org/abs/1912.04977.

[4] M. M. Amiri et al., "Federated Learning Over Wireless Fading Channels," IEEE Trans. on Wireless Comm., vol. 19, no. 5, pp. 3546-3557, May 2020.

[5] J. A Daily, et al., "Gossipgrad: Scalable deep learning using gossip communication based asynchronous gradient descent," [Online]. Available: https://arxiv.org/abs/1803.05880.

[6] S. Savazzi, et al. "Federated Learning with Cooperating Devices: A Consensus Approach for Massive IoT Networks," IEEE Internet of Things Journal, vol. 7, no. 5, pp. 4641-4654, May 2020. [Online]. Available: https://tinyurl.com/y2kr7rw5.

[7] A. H. Sayed, et al., "Diffusion Strategies for Adaptation and Learning over Networks: An Examination of Distributed Strategies and Network Behavior," IEEE Signal Proc. Mag., vol. 30, no. 3, pp.155-171, 2013.

[8] A. Elgabli, et al., "GADMM: Fast and Communication Efficient Framework for Distributed Machine Learning," Journal of Machine Learning Research, vol. 21 pp. 1-390, 2020.

[9] H. Xing, et al. "Decentralized Federated Learning via SGD over Wireless D2D Networks," Proc. IEEE SPAWC, 2020.

[10] S. Wang, et al., "Adaptive Federated Learning in Resource Constrained Edge Computing Systems," IEEE Journal on Sel. Areas in Comm., vol. 37, no. 6, pp. 1205-1221, June 2019.

[11] H. Kim, et al. "Blockchained On-Device Federated Learning," IEEE Communications Letters, June 2019.

[12] M. Chen, et al. "Performance Optimization of Federated Learning over Wireless Networks," Proc. IEEE GLOBECOM, Waikoloa, HI, USA, pp. $1-6,2019$. 
[13] R. Nassif, et al. "Multitask Learning Over Graphs: An Approach for Distributed, Streaming Machine Learning," IEEE Signal Proc. Mag., vol. 37, no. 3, pp. 14-25, May 2020.

[14] M. Brambilla, et al., "Augmenting Vehicle Localization by Cooperative Sensing of the Driving Environment: Insight on Data Association in Urban Traffic Scenarios," IEEE Trans. on Intelligent Transportation Systems, vol. 21, no. 4, pp. 1646-1663, April 2020.

[15] S. Kianoush, et al. "A Multisensory Edge-Cloud Platform for Opportunistic Radio Sensing in Cobot Environments," IEEE Internet of Things Journal, vol. 8, no. 2, pp. 1154-1168, January 2021.

Stefano Savazzi is Researcher at the Consiglio Nazionale delle Ricerche (CNR) with the Institute of Electronics, Computer and Telecommunication Engineering (IEIIT). He was visiting researcher with the Uppsala University (2005) and the UCSD, San Diego (2009). He co-authored over 100 scientific publications. Research interests include signal processing, learning and networking design aspects for the Internet of Things and beyond 5G. He is the recipient of the Dimitris N. Chorafas Foundation Award.

Monica Nicoli is an Associate Professor at Politecnico di Milano, with the Department of Management, Economics and Industrial Engineering. She was visiting researcher with ENI-Agip (Italy) in 1998-1999 and with Uppsala University (Sweden) in 2001. She co-authored over 100 scientific publications. Her research interests are in the area of statistical signal processing, with focus on wireless communications, localization and smart mobility. She is a recipient of the 1999 Marisa Bellisario Award, and a co-recipient of the 2014 IET Intelligent Transport Systems and the 2018 IEEE Statistical Signal Processing Workshop Best Paper Awards.
Mehdi Bennis is an Associate Professor at the Centre for Wireless Communications, University of Oulu, Finland, an Academy of Finland Research Fellow and head of the intelligent connectivity and networks/systems group (ICON). His main research interests are in radio resource management, heterogeneous networks, game theory and machine learning in 5G networks and beyond. He was the recipient of several prestigious awards including the 2015 Fred W. Ellersick Prize from the IEEE Communications Society, the 2016 Best Tutorial Prize from the IEEE Communications Society, the 2017 EURASIP Best paper Award for the Journal of Wireless Communications and Networks, the all-University of Oulu award for research and the 2019 IEEE ComSoc Radio Communications Committee Early Achievement Award.

Sanaz Kianoush is a Postdoctoral researcher at the IEIIT institute of the CNR since November 2014. Visiting researcher at Aalto University in 2018 , lecturer at Azad University Sary (Iran) in 2008. Her research interests include statistical signal processing, machine learning in communication systems, device-free radio localization.

Luca Barbieri is currently a Ph.D. candidate in Information Technology at DEIB department, Politecnico di Milano. His current research interests focus on machine learning and localization techniques for vehicular and industrial networks 